\title{
A Stochastic Approach to Occupant Pre-movement in Fires
}

\author{
JAMIE VISTNES ${ }^{1}$, STEPHEN J GRUBITS ${ }^{1}$, and YAPING HE ${ }^{2}$ \\ ${ }^{1}$ Stephen Grubits \& Associates \\ P.O. Box N522, Grosvenor Place \\ NSW, 1220 Australia \\ ${ }^{2}$ University of Western Sydney \\ Locked Bag 1797, Penrith South DC \\ NSW, 1797 Australia
}

\begin{abstract}
A stochastic model has been developed to estimate the pre-movement time of occupants in a fire situation. The model quantifies the response and delay periods using probability distribution functions and Monte Carlo simulation techniques. The model takes into account both the occupants and building characteristics. The times spent on various delay actions by the occupant are treated as random variables with prescribed distribution functions. When a delay action is related to a quantifiable occupant or building characteristic, the associated parameter is also assumed to be a random parameter and the appropriate governing distribution is assigned. The model produces, for a given building design and occupant group, the probability distribution of pre-movement time and the related parameters. The results of simulation runs are presented in this paper. The results correlate reasonably well with the data obtained from the literature. Sensitivity studies are also carried out and the regression and correlation are analysed.
\end{abstract}

KEYWORDS: human behaviour, pre-movement, risk assessment, stochastic

\section{INTRODUCTION}

The evaluation of the required safe egress time is an important step in the performance based fire safety engineering design [1]. This time parameter is related to the design of building fire safety features as well as occupant characteristics and is often treated as having three time components, namely, detection time, pre-movement time and movement time. The evaluation of pre-movement time is the focus of the current paper.

The field of occupant pre-movement in fire has been the subject of much research over the past century [2]. A number of quantification methods can be found in the SFPE Handbook [3,4] and Fire Engineering Design Guide [1]. These methods are based on the specification of overall pre-movement times for different occupancies and warning systems, and neglect the influence of building characteristics. Another method was proposed by Sime [5] which provides baseline estimates of response time based upon types of warning systems, however no further specific building characteristics are utilised. The method also focuses on providing weighting factors to these baseline estimates which are defined by a number of characteristics based upon the occupants.

Models such as CESARE Risk [6], CRISP [7] and FIERAsystem [8] also provide a means of estimating occupant pre-movement time. Although these models incorporate the cues provided and the surrounding environment to determine occupant response and delay, the quantification of these periods is quite limited and simplistic. The models adopt to some extent a probabilistic methodology in determining the likelihood of 
responses and actions, however when it comes to specifying times for these actions, most models generally revert to using discrete values, except for CESARE Risk and CRISP which allow for limited distributions for the response period to be used. Due to the limited use of probability distributions and the simplistic treatment of actions using discrete values, it is considered that these models would not sufficiently account for the varying levels of abilities of occupants within an entire population of an occupancy. CESARE Risk and FIERAsystem also only allow for a quite limited range of delay actions to occur.

A stochastic approach was proposed by He et al. [9], in which the building evacuation process is treated as a counting process that follows the Poisson distribution. The determination of the sole parameter of the Poisson distribution, namely, the population flow rate, was based on the conventional deterministic approach and therefore, has limited scope.

The objective of this paper is to develop a stochastic occupant pre-movement model that will more appropriately estimate the likely pre-movement times of occupants in a fire situation. The paper provides an outline of a modelling approach utilising stochastic methodology to incorporate the activities undertaken by occupants in response to fire and identifies where further work and data is required.

\section{METHODOLOGY}

\section{Stochastic Modelling}

Any dynamic system that involves random parameters or parameters that are too complicated to be described by deterministic means can be regarded as a stochastic system and the associated process a stochastic process [10]. Human behaviour in fire emergencies is a very complicated phenomenon. The parameters used to describe this phenomenon appear to have a large degree of uncertainty or randomness. A parameter of interest in fire safety engineering assessment is the required safe egress time which is usually decomposed into detection time, pre-movement time and movement time [11]. This time parameter is a result of collective actions of many individuals. Yet, each individual does not have a set or predetermined evacuation time, let alone the ensemble required safe egress time. The situation is best handled with stochastic modelling.

A Monte Carlo simulation of a large number of occupant pre-movement scenarios was adopted as a basis for the stochastic model. In order to ensure an adequate representation of low probability events, a Latin hypercube sampling algorithm was adopted. This method is a stratified sampling technique in which the probability distribution is divided into a number of regions (depending upon the number of iterations chosen), in which values are randomly selected from within each region. This therefore ensures that the distribution is effectively sampled [12].

\section{The Pre-movement Model}

The occupant pre-movement model represents the events that follow the occurrence of cues up until the initiation of movement to a safe place [11], i.e., the response and delay periods. These time periods are either random variables themselves or functions of other random variables. 
Response Period - The response period involves interpretation of the cue as a cause for action. It is a decision process where occupants decide whether to respond to the cue or ignore it. Therefore, since the only action involved in this period is the decision of whether to respond to the cue or not, the quantification of this period involves specifying a time for the interpretation and decision process to occur. It is important to note that every occupant will undertake this process during the pre-movement period.

Delay Period - Upon recognising the cue as a cause for action, occupants generally undertake a number of actions prior to evacuating. These actions are labelled delay actions and constitute the delay period. The number, type and duration of these actions may vary from occupant to occupant. Therefore, the quantification of the delay period is more extensive and involves quantifying the range of delay actions that occur. This quantification involves predicting which actions occur (identification of the actions), how often they occur (the probability of occurrence), and the time the occupant takes to undertake each action (the duration of the actions). Unlike the interpretation or decision process in the response period, not all occupants will undertake all (or any) of the identified delay actions in the delay period.

As occupants would have different priorities in a fire situation as well as varying levels of ability and commitment, the response times, types of actions and duration of actions will differ from occupant to occupant. Therefore, the model needs to cater for this differing level of pre-movement activities that are undertaken so that an adequate representation of the pre-movement time of the entire occupancy may be obtained.

By specifying a distribution for the response time and times for actions to be completed, the varying levels of response due to different occupant characteristics may be modelled. Associating probabilities of occurrence with the relevant delay actions would model the fact that occupants may undertake any number of actions and any type of action.

\section{Implementation of the Model}

The stochastic model is implemented using the Microsoft Excel add-in “@RISK” [13]. After the model is defined in @RISK and the appropriate inputs and outputs are defined, a simulation of the model is then run which undertakes a number of iterations of the model. Each iteration takes a sample from each of the probability distribution functions and all expressions are calculated, including the pre-movement time output. Each iteration would represent one "occupant" going through the pre-movement process. The model will effectively simulate the pre-movement activities of an entire population, with varying actions and times, and provide an estimate of the pre-movement time for the entire population. The end result is a numerical description of the probability density distribution of the pre-movement time for a given building design.

\section{MODEL PARAMETERS}

\section{Response Time}

The response period involves the interpretation of the cue as a cause for evacuation. The response time has been found to be largely influenced by the type of warning provided to the occupant [14]. Two types of warning system are included in the current model, an alarm system with a bell or sounder as a warning, and an informative warning system such as an Early Warning and Intercommunication System (EWIS). 
The distribution for the response time could not be explicitly obtained from the data currently available, therefore observations on the overall pre-movement time were considered. As described in [5], the general trend of the distribution of times involved in occupant pre-movement suggest a log-normal distribution, and therefore this distribution has been adopted in representing the times for the response period.

A log-normal distribution requires an average and standard deviation to be specified. The average response time of occupants to each of the warning systems has been obtained from experimental studies of evacuations in different building types by Purser [15]. For a bell or sounder system the value is 60 seconds and for informative systems 40 seconds. Previous estimates of occupant pre-movement time based upon current research have specified ranges of likely pre-movement times [5]. These ranges of times have been considered in the specification of the standard deviation of the response time distribution, as well as the specification of the upper and lower limits of the distribution.

\section{Delay Time}

\section{Identification of Delay Actions}

The types of delay actions occupants undertake in the period between deciding to respond to the cue and beginning to evacuate are dependant upon the individuals' characteristics, the type of occupancy and the state of the occupants' environment. There could be a large number of different delay actions that are carried out by occupants during the delay period. A number of studies on occupant response in fires have identified the types of delay actions $[3,7,8,16,17,18,19]$. The typical occupancies involved in these studies include office, retail, industrial, institutions (hospitals and schools), hotels and residential. The most common delay actions identified were:

$$
\begin{array}{lll}
\text { - Notify others; } & \text { - Telephoned others; } & \text { - Got dressed; } \\
\text { - Investigate/search for fire; } & \text { - Close/open doors/windows; } & \text { - Woke up; and } \\
\text { - Call Fire Brigade; } & \text { - Raise Alarm; } & \text { - Fight fire. } \\
\text { - Inaction; } & \text { - Shut down equipment; } & \\
\text { - Collect belongings; } & \text { - Rescue; }
\end{array}
$$

The actions "got dressed" and "woke up" are considered only appropriate to residential occupancies or occupancies that accommodate sleeping occupants. Therefore two models were set up, one for "residential" occupancies which included the residential occupancy actions, and one for "generic" occupancies which excluded the actions specific to residential occupancies.

It should also be noted that the action of "raise alarm" was only common in areas where occupants could see the fire and when the automatic alarm was not activated. This was shown in [19] where the likelihood of occupants activating the alarm in response to the cue of an automatic alarm sounding is virtually nil. The action "fight fire" was identified as being common but is not incorporated in the model at present, as it constitutes an action whose time depends on the fire characteristics as well as the occupant characteristics.

\section{Probabilities for Delay Actions}

As the delay actions undertaken will vary from occupant to occupant, the delay actions were assigned probabilities of occurrence to define the frequency in which they will be 
undertaken. The model was developed to incorporate the total percentages of occupants undertaking the specific delay actions at some stage throughout the delay period.

The percentage of occupants undertaking certain delay actions at some stage during the delay period has been identified in a number of studies $[8,17,18]$. For studies where the data has been provided for occupants undertaking certain delay activities as subsequent actions [3], the percentages stated have been collated to obtain the total percentage of occupants undertaking the specific delay actions during the delay period. All these percentages obtained were then combined to provide an average estimate of the total percentages of occupants undertaking the delay actions in the delay period, which are shown in Table 1 . These delay actions were observed in occupancies such as office, retail, industrial, institutions (hospitals and schools), hotels and residential.

These percentages were used as discrete probabilities associated with each delay action to determine whether or not a particular delay action was undertaken by an occupant during each iteration. The model did not set any limits on the number of delay actions undertaken by an occupant, and therefore the theoretical maximum number of delay actions undertaken is equal to the number of delay actions specified. The number of delay actions undertaken by an occupant in any one iteration of the model is dependant on how many delay actions were "selected" as being undertaken during that iteration based upon the discrete probabilities specified.

\section{Definitions and Duration of Delay Actions}

Numerous studies have indicated that various building and occupant characteristics affect occupant pre-movement $[4,5,20,21]$. The definitions of each of the delay actions used to quantify the delay period in the stochastic model are summarised in Table 1 . The parameters used in the definition of the delay actions are further described below. Some delay actions involve movement of building occupants, such as travel to telephone location and travel to notify others. The associated travel time is distinguished in the current model. Let $a_{i}$ denote the time taken for action $i$, the total delay time period, $t_{i}$, for action $i$ is generally expressed as shown in Eq. 1 where $d_{i}$ is travel distance and $v$ travel speed.

$$
t_{i}=\frac{d_{i}}{v}+a_{i}
$$

The former is related to the building characteristics and the latter the occupant. All parameters on the right hand side of Eq. 1 are random variables and their treatment in the model are illustrated in the following sections. If an action does not involve travel, then the travel distance for that act is set to zero. The total pre-movement time, $t_{p}$, is the response time, $t_{r}$, plus the summation of all delay time components as shown in Eq. 2.

$$
t_{p}=t_{r}+\sum_{i}^{N} t_{i}
$$


Table 1. Definitions of delay actions and associated percentages of being undertaken.

\begin{tabular}{|c|c|c|c|c|}
\hline $\begin{array}{l}\text { ID } \\
\text { No. }\end{array}$ & Delay Action & Description & Delay Time & $\begin{array}{c}\text { Total } \\
\%\end{array}$ \\
\hline 1 & Notify others & $\begin{array}{l}\text { Time to notify other } \\
\text { occupants (within } \\
\text { immediate area) }\end{array}$ & $\begin{array}{l}\text { Time to travel within } \\
\text { immediate area + time } \\
\text { to talk or notify }\end{array}$ & 25.3 \\
\hline 2 & $\begin{array}{l}\text { Investigate/ } \\
\text { search for fire }\end{array}$ & $\begin{array}{l}\text { Time to search for the } \\
\text { fire (within an area } \\
\text { familiar to the } \\
\text { occupant) }\end{array}$ & $\begin{array}{l}\text { Time to travel within } \\
\text { familiar area }\end{array}$ & 12.1 \\
\hline 3 & $\begin{array}{l}\text { Call Fire } \\
\text { Brigade }\end{array}$ & $\begin{array}{l}\text { Time to get to phone } \\
\text { and call the fire brigade }\end{array}$ & $\begin{array}{l}\text { Time to travel half } \\
\text { distance within } \\
\text { immediate area }+ \text { time } \\
\text { to talk on phone to } \\
\text { fire brigade }\end{array}$ & 32.8 \\
\hline 4 & Inaction & No active action & Idling time & 11.8 \\
\hline 5 & $\begin{array}{l}\text { Collect } \\
\text { belongings }\end{array}$ & $\begin{array}{l}\text { Time to travel to } \\
\text { belongings and collect }\end{array}$ & $\begin{array}{l}\text { Time to travel within } \\
\text { immediate area }+ \text { time } \\
\text { to collect belongings }\end{array}$ & 12.6 \\
\hline 6 & $\begin{array}{l}\text { Telephoned } \\
\text { others }\end{array}$ & $\begin{array}{l}\text { Time to get to a phone } \\
\text { and call others (such as } \\
\text { relatives) }\end{array}$ & $\begin{array}{l}\text { Time to travel half } \\
\text { distance within } \\
\text { immediate area }+ \text { time } \\
\text { to talk on phone to } \\
\text { others }\end{array}$ & 5.1 \\
\hline 7 & $\begin{array}{l}\text { Close/open } \\
\text { doors/windows }\end{array}$ & $\begin{array}{l}\text { Time to travel to } \\
\text { opening and open or } \\
\text { close it }\end{array}$ & $\begin{array}{l}\text { Time to travel within } \\
\text { immediate area + time } \\
\text { to close or open }\end{array}$ & 9.3 \\
\hline 8 & $\begin{array}{l}\text { Shut down } \\
\text { equipment }\end{array}$ & $\begin{array}{l}\text { Time to travel to } \\
\text { equipment and shut it } \\
\text { down }\end{array}$ & $\begin{array}{l}\text { Time to travel within } \\
\text { immediate area + time } \\
\text { to shut down }\end{array}$ & 1.5 \\
\hline 9 & Rescue & $\begin{array}{l}\text { Time to travel to } \\
\text { occupant \& assist } \\
\text { rescue }\end{array}$ & $\begin{array}{l}\text { Time to travel within } \\
\text { immediate area + time } \\
\text { to assist in rescue }\end{array}$ & 12.4 \\
\hline 10 & Got dressed & Time to put clothes on & Dressing time & 48.5 \\
\hline 11 & Woke up & Time to wake up & Wake up time & 3.8 \\
\hline
\end{tabular}

${ }^{\mathrm{a}}$ ID number is purely for identification purpose. It does not indicate action sequence.

\section{Building Characteristics}

The building characteristics utilised in the stochastic model are the type of warning system installed in the building and the layout of the building, i.e., the travel distances occupants are required to travel. The warning system is used to define the likely response times as discussed previously. The building layout has an impact upon the distances occupants are required to travel, in order that they may undertake certain delay actions.

Two travel distances are specified in the definitions used to quantify the delay actions. These are the travel distance within the immediate area (e.g., the occupants' office or sole 
occupancy unit) and the travel distance within the familiar area (e.g., the occupants' floor). The actual travel distance by any occupant depends on the initial location of the occupant and is a random variable. It is assumed that the probability density distribution function that governs this random variable is a uniform distribution, as it is equally likely that an occupant may be anywhere within the area.

Some actions, such as investigation and notifying others, may involve travelling back and forth within a defined area. To account for multiple travelling, the maximum travel distance within the area is specified. The detailed description of the strategy for determining the maximum travel distance in particular building layouts can be found in [22]. For the purposes of the simulations run in this paper, the maximum travel distance within the immediate area has been taken as $160 \mathrm{~m}$ and the maximum travel distance within the familiar area has been taken as $240 \mathrm{~m}$.

\section{Occupant Characteristics}

The occupant characteristics used in the quantification of the pre-movement time are mobility, location and alertness/awareness. The occupants location is represented in the travel distances outlined above. The occupants alertness and awareness is characterised in the response time specified previously. In order to quantify the time involved in travelling the distances outlined above, the occupants travel speed, which is considered to be representative of the occupants mobility, needs to be specified. The distribution used for travel speed in the stochastic model is obtained from observations of a population made by Fruin [23]. These observations have been correlated to a truncated normal distribution for use in the model.

\section{Time Periods $\left(a_{i}\right)$}

Studies of pre-movement times have shown that in relation to their probability distribution, the mode, or most common occurring time, is always to the left of the mean, resulting in a skewed distribution [24]. It has also been indicated that the overall occupant pre-movement times when assessed in a probabilistic sense may have a log-normal distribution [5]. This is due to the majority of the population responding within a reasonable time, but the distribution having a long tail to account for the "stragglers" who take much longer to complete tasks. It is therefore postulated that the times taken by the activities making up the overall pre-movement time (such as the delay actions) would also have similar distributions.

A log-normal distribution as described previously has been adopted. The parameters specified for each time constant are shown in Table 2. The mean and standard deviation have been selected to create a log-normal distribution between values that are considered likely to occur. These parameters provide for a log-normal distribution skewed to the left with the mode to the left of the mean (as observed in the literature for the trends in premovement time).

It should be noted that if the user has more specific information relating to the occupancy, such as their ability to undertake certain actions, these time estimates may be revised to represent this level of ability in the model. 
Table 2. Parameters for time constant distributions.

\begin{tabular}{|c|l|c|c|}
\hline $\begin{array}{c}\text { Action ID } \\
\text { Number }\end{array}$ & \multicolumn{1}{|c|}{ Delay Action } & $\begin{array}{c}\text { Mean } \\
\text { (s) }\end{array}$ & $\begin{array}{c}\text { Standard Deviation } \\
\text { (s) }\end{array}$ \\
\hline 1 & Notify others & 10 & 3 \\
\hline 2 & Call Fire Brigade & 30 & 9 \\
\hline 3 & Inaction & 60 & 18 \\
\hline 4 & Collect belongings & 30 & 9 \\
\hline 5 & Telephoned others & 30 & 9 \\
\hline 6 & Close/open doors/windows & 5 & 1.5 \\
\hline 7 & Shut down equipment & 20 & 6 \\
\hline 8 & Rescue & 30 & 9 \\
\hline 9 & Got dressed & 60 & 18 \\
\hline 10 & Woke up & 60 & 18 \\
\hline
\end{tabular}

\section{RESULTS AND DISCUSSION}

\section{Comparisons with Current Research}

One source of literature proved particularly useful for comparison with the results of the stochastic model. Proulx and Fahy [25] collated data from a number of evacuation studies with the intent to begin the creation of a database on occupant pre-movement time. This data was organised such that it provided the mean, minimum, maximum, median and quartiles for each study listed in the data set. The data was separated according to occupancies in order that comparisons could be made with the stochastic model. The data on office and retail occupancies was collected and the values averaged. The resulting cumulative probability distribution function shown in Eq. 3 is compared with that of the model "generic" simulations in Fig. 1.

$$
P(t)=\operatorname{Prob}\left\{t_{p} \leq t\right\}
$$

The model correlates quite well with the research data up until the third quartile. The maximums differ to some extent, however this end of the distribution is associated with low probabilities and therefore the values in these regions are not as significant. The probability density distribution function (PDDF) for the overall pre-movement time obtained from the stochastic model is shown in Fig. 2.

Similarly, the data on residential and hotel occupancies was collected and averaged, with the results compared to the stochastic model for "residential" simulations shown in Fig. 3. The model correlates quite well with the research data up until the first quartile. Past this, the stochastic model predicts times much less than those observed in the literature. However, the research data included buildings with no alarm system, whereas the stochastic model presented in this paper assumes an alarm system. Therefore, the data for residential studies that incorporated buildings with alarm systems was extracted and compared with the model prediction in Fig. 4. The results of the model correlate well with the findings from Proulx and Fahy. 


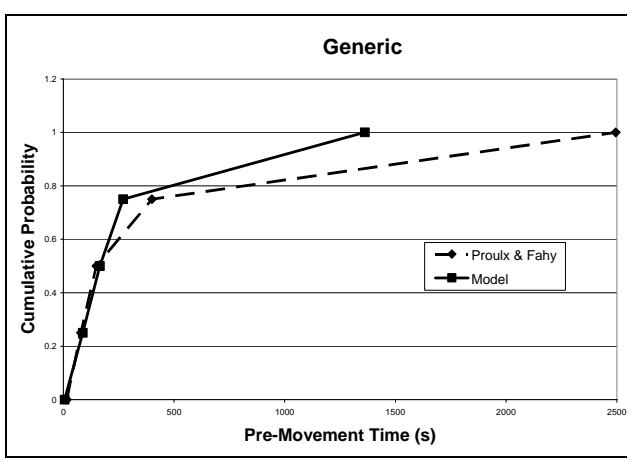

Fig. 1. Comparison of stochastic model with findings from Proulx \& Fahy [25] ("Generic” occupancy).

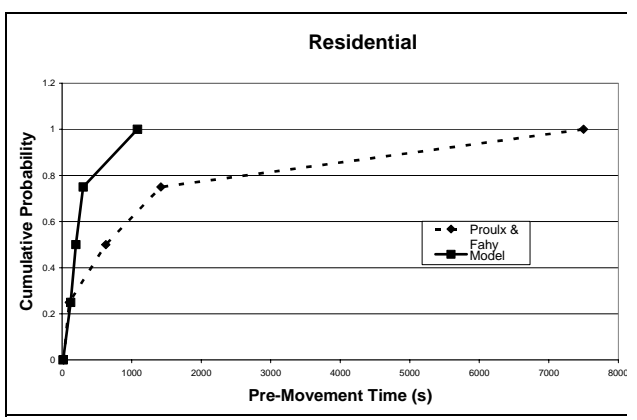

Fig. 3. Comparison of stochastic model with findings from Proulx \& Fahy [25]

("Residential” occupancy).

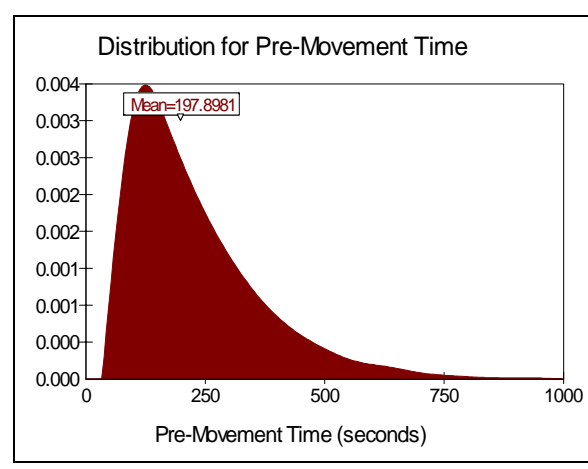

Fig. 2. PDDF for the overall premovement time obtained from the "generic" stochastic model.

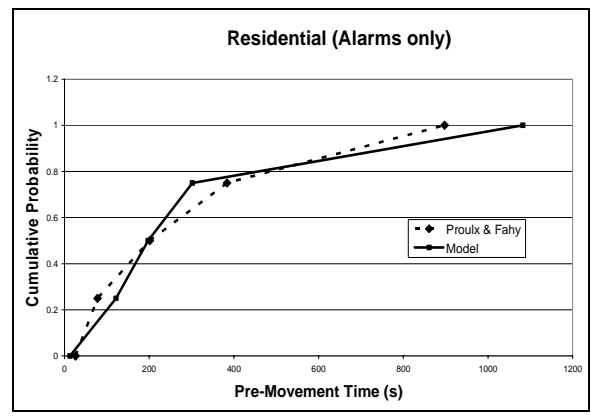

Fig. 4. Comparison of stochastic model with findings from Proulx \& Fahy

[25] ("Residential” occupancy with alarms only).

\section{Sensitivities}

The sensitivity to the number of iterations chosen for the simulation was assessed using the "generic" model. Simulations using one thousand, one hundred thousand and one million iterations were compared to the simulation using ten thousand iterations. The results show that the distribution obtained from the simulation using one hundred thousand and one million iterations are not significantly different from the simulation using ten thousand, with the differences being approximately $0.5 \%$ or less. Therefore, it is concluded that ten thousand iterations is sufficient for these simulations.

A simulation of the "generic" model with ten thousand iterations was run, doubling the means and standard deviations for the probability density distribution functions for the time constants assumed. The time constants doubled in this assessment were only those values where there was limited data and the values were assumed. The results show differences in the key parameters of up to $20 \%$. However, the doubling of these time constants resulted in durations of approximately four minutes or more for one delay action. This is considered excessive compared to the times observed in the literature. Although, this sensitivity study is useful in that it shows that a $100 \%$ increase in these time constants increases the overall pre-movement time by no more than $20 \%$. 
Regression relates to the sensitivity to the input variables. A "tornado graph" of regression for pre-movement time for the model with the residential actions included is shown in Fig. 5. On this tornado graph, an increase in one standard deviation of the input variable relates to an increase in the pre-movement time of the standard deviation multiplied by the regression coefficient. All inputs are to the right of the tornado graph indicating that an increase in their value will result in a positive increase in the premovement time, as would be expected.

Correlation coefficients are calculated between the outputs and each set of sampled inputs. A correlation coefficient of 1 indicates that the input variable is highly correlated with the output, whereas a correlation coefficient of -1 indicates an inverse correlation. A tornado graph of the correlation coefficients for the "residential" model is shown in Fig. 6. All correlation coefficients are positive for the input values, suggesting that when the times for the inputs are high, so is the time for the pre-movement time output.

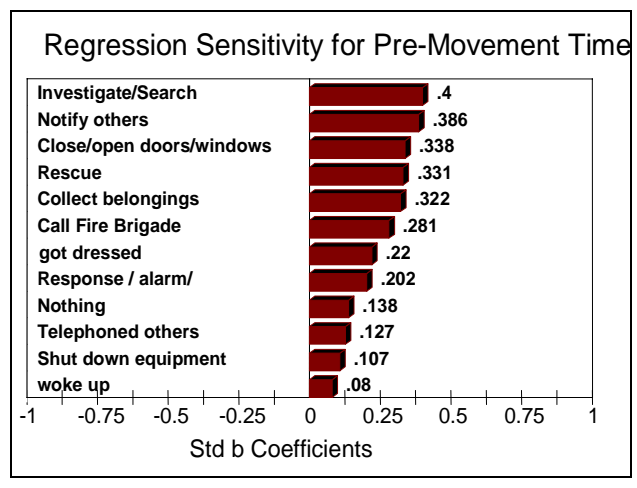

Fig. 5. Regression Sensitivity for PreMovement Time (residential).

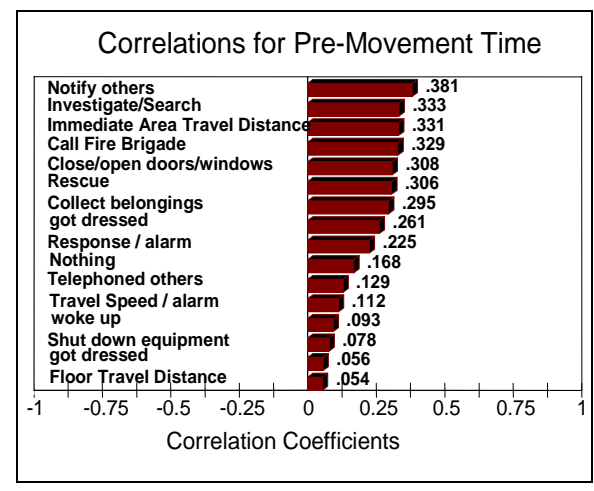

Fig. 6. Correlation for Pre-Movement Time (residential).

\section{CONCLUSION}

A stochastic model has been developed to estimate the pre-movement time of occupants in a fire situation. The model quantifies the time components associated with response and delay actions using probability distribution functions and stochastic modelling techniques. The model produces a probability density distribution function for premovement time, which can be used for risk based engineering assessment. Although the data obtained from current research is relatively limited, the results of the stochastic model using this data correlate well with current observations of occupant pre-movement times. Due to the limited treatment of variables in other pre-movement models, it is considered the stochastic model proposed in this paper assesses in more depth the response and delay periods which make up the pre-movement period.

The model regards the following phenomena as random processes: the response time, the delay actions undertaken by an occupant, the location and travel speed of the occupants, and the time spent on delaying actions. Both the building characteristics and the occupant characteristics are incorporated into the model. The model is capable of addressing the variation and uncertainty in the components that make up the pre-movement time. In fact, the model is capable of dealing with the movement time period as well.

The output distribution of pre-movement time enables the stochastic model to provide an assessment of the entire population involved in the pre-movement period prior to 
evacuation. This output gives the opportunity to gain an appreciation of the range of premovement times possible within a given occupancy. The outputs from the stochastic model would also be useful for input into other probabilistic models. The stochastic model presented in the current paper is essentially a Markovian type where the memory of the modelled subject, or the order of the actions, is not taken into account. The effect of the memory requires further investigation and will be part of future studies.

Due to the lack of information in the literature in regard to the duration of the delay actions, some of the input parameters used in the described simulation study were somewhat arbitrarily selected. It is considered that if further input data can be obtained, the stochastic model can be used to produce a better estimate of probability density distributions for occupant pre-movement times. Therefore, some investigation into the times of the delay actions and the nature of these actions (i.e., how occupants undertake these actions) would be desirable. The majority of the data obtained was provided from studies where the occupancies were mixed or limited. It would therefore be desirable to gain data for specific occupancies and for a wider range of occupancies.

\section{ACKNOLEDGEMENT}

The authors would like to thank Dr Caird Ramsay for his guidance and valuable suggestions during the course of the research.

\section{REFERENCES}

[1] Buchannan, A., "Fire Engineering Design Guide," Centre for Advanced Engineering, University of Canterbury, 1994.

[2] Bryan, J.L., “A Selected Historical Review of Human Behaviour in Fire,” Fire Protection Engineering Number 16, SFPE, Fall, 2002.

[3] Bryan, J.L., "Behavioural Response to Fire and Smoke," Chapter 3-12, SFPE Handbook of Fire Protection Engineering - National Fire Protection Association - 3rd Edition, 2002.

[4] Proulx, G., "Movement of People: The Evacuation Timing," Chapter 3-13, SFPE Handbook of Fire Protection Engineering - National Fire Protection Association - 3rd Edition, 2002.

[5] Sime, J.D., “Assessing Occupant Response Time: A Key Issue for Fire Engineering,” R. Barham, 1996 (ed), Fire and Emergency Planning: Research and applications, London, 1996.

[6] Thomas, I.R., "The Development of CESARE Risk: A Fire-Risk CostAssessment Program,” Fire Protection Engineering Number 19, Summer, 2003.

[7] Fraser-Mitchell, J.N., "Modelling Human Behaviour within the Fire Risk Assessment Tool "CRISP"," Human Behaviour in Fire, 1st International Symposium, 1998, Belfast.

[8] Raboud, D.W., Benichou, N., Kashef, A., Proulx, G., and Hadjisophocleous, G.V., "FIERAsystem Occupant Response (OCRM) and Occupant Evacuation (OEVM) Models Theory Report,” NRC CNRC, May, 2002. 
[9] He, Y., Horasan, M., Taylor, P., and Ramsay, C., 2003, "Stochastic Modelling for Risk Assessment," Proceedings of the 7th International Symposium on Fire Safety Science, International Association of Fire Safety Science, June 14-20, 2002, Worcester Polytechnic Institute, USA, pp. 333-344.

[10] Pugachev, V.S., and Sinitsyn, I.N., 2001, "Stochastic Systems," World Scientific, New Jersey.

[11] Australian Building Codes Board, “Fire Safety Engineering Guidelines,” Edition 2001.

[12] Palisade Corporation, “Guide to Using @RISK,” Version 4, USA, 2000.

[13] Palisade Corporation, “@RISK,” Version 4, USA, 2000.

[14] Proulx, G., and Sime, J.D., "To Prevent 'Panic' in an Underground Emergency: Why Not Tell People the Truth?" Proceedings of the Third International Symposium on Fire Safety Science, London, 1991.

[15] Purser, D.A., "Quantification of Behaviour for Engineering Design Standards and Escape Time Calculations,” Human Behaviour in Fire, 1st International Symposium, 1998, Belfast.

[16] Canter, D., “Fires and Human Behaviour,” Wiley, USA, 1980.

[17] Proulx, G., and Fahy, R., "Human Behaviour in the World Trade Centre Evacuation," Fire Safety Science - Proceedings of the Fifth International Symposium, 1997.

[18] Proulx, G., “Occupant Response During a Residential Highrise Fire,” NRC CNRC, 1999.

[19] Saunders, W.L., "Occupant Decision Making in Office Building Fire Emergencies," Fire Safety Science - Proceedings of the Fifth International Symposium, 1997.

[20] Proulx, G., “Occupant Behaviour and Evacuation,” NRC CNRC, 2001.

[21] Proulx, G., and Fahy, R.F., "The Time Delay to Start Evacuation: Review of Five Case Studies," Fire Safety Science - Proceedings of the Fifth International Symposium, 1997.

[22] Vistnes, J., 2004, “A Stochastic Approach to Occupant Pre-Movement in Fires,” Masters dissertation, University of Western Sydney, Australia.

[23] Fruin, J.J., “Pedestrian Planning and Design,” Elevator World, USA, 1987.

[24] MacLennan, H.A., Regan, M.A. and Ware, R., "An Engineering Model for the Estimation of Occupant Pre-movement and or Response Times and the Probability of Their Occurrence," Human Behaviour in Fire - Proceedings of the First International Symposium, Shields, T.J. (Editor), August 31 September 2, 1998, University of Ulster, pp.13-29.

[25] Proulx, G., and Fahy, R., "Toward Creating a Database on Delay Times to Start Evacuation and Walking Speeds for Use in Evacuation Modelling,” NRC CNRC, 2001. 